\title{
The House Dust Mite Major Allergen Der p 23 Displays O-Glycan-Independent IgE Reactivities but No Chitin-Binding Activity
}

\author{
Wai Tuck Soh $^{\mathrm{a}}$ Maxime Le Mignon ${ }^{\mathrm{h}}$ Narissara Suratannon ${ }^{\mathrm{b}}$

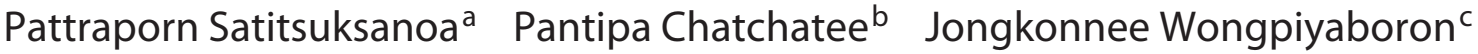 \\ Mukda Vangveravong ${ }^{d}$ Ticha Rerkpattanapipat ${ }^{\mathrm{e}}$ Atik Sangasapaviliya $^{\mathrm{f}}$ \\ Emmanuel Nony $^{\mathrm{h}}$ Surapon Piboonpocanung ${ }^{\mathrm{K}}$ Kiat Ruxrungtham ${ }^{\mathrm{a}}$ \\ Alain Jacquet $^{\mathrm{a}}$ Mite Allergy Research Cohort (MARC) Study Team \\ ${ }^{a}$ Division of Allergy and Clinical Immunology, Department of Medicine and Chula Vaccine Research Center, \\ ${ }^{b}$ Division of Allergy and Immunology, Department of Pediatrics, and ${ }^{\mathrm{C} C e n t e r ~ o f ~ E x c e l l e n c e ~ i n ~ I m m u n o l o g y ~ a n d ~}$ \\ Immune Mediated Diseases, Division of Immunology, Department of Microbiology, Faculty of Medicine, \\ Chulalongkorn University, ${ }^{\mathrm{d}}$ Allergy, Immunology and Rheumatology Unit, Queen Sirikit National Institute of \\ Child Health, Department of Medical Services, Ministry of Public Health, and 'Division of Allergy, Immunology and \\ Rheumatology, Department of Medicine, Faculty of Medicine, Ramathibodi Hospital, Mahidol University, and \\ fDivision of Allergy and Clinical Immunology, Department of Medicine, Phramongkutklao Hospital, Bangkok, and \\ gInstitute of Molecular Biosciences, Mahidol University, Nakhon Pathom, Thailand; h Stallergenes SA, Antony, France
}

\section{Key Words}

Allergen $\cdot$ Chitin $\cdot$ Der p $23 \cdot$ House dust mite $\cdot \lg$ E reactivity . O-glycosylation

\begin{abstract}
Background: The in-depth characterization of the recently identified house dust mite (HDM) major allergen Der p 23 requires the production of its recombinant counterpart because the natural allergen is poorly extractable from fecal pellets. This study aimed to provide a detailed physicochemical characterization of recombinant Der $p 23$ ( $r$ Der $p$ 23) as well as to investigate its IgE reactivity in a cohort of HDM-allergic patients from Thailand. Methods: Purified rDer p 23, secreted from recombinant Pichia pastoris, was characterized by mass spectrometry and circular dichroism analyses as well as for its chitin-binding activity. The lgE-
\end{abstract}

binding frequency and allergenicity of Der $\mathrm{p} 23$ were determined by ELISA and RBL-SX38 degranulation assays, respectively. Results: Purified intact rDer p 23 carried O-mannosylation and mainly adopted a random coil structure. Polyclonal antibodies to rDer p 23 can detect the corresponding natural allergen (nDer $\mathrm{p} 23$ ) in aqueous fecal pellet extracts, suggesting that both forms of Der $p 23$ share common B-cell epitopes. Despite its homologies with chitin-binding proteins, both natural Der p 23 and rDer p 23 were unable to interact in vitro with chitin matrices. Of 222 Thai HDM-allergic patients tested, 54\% displayed Der $p$ 23-specific IgE responses. Finally, the allergenicity of rDer $p$ 23 was confirmed by the degranulation of rat basophil leukemia cells. Conclusion: Our findings highlighted impor-

The members of the MARC Study Team are listed in the Appendix.

\section{KARGER 125}

(c) 2016 S. Karger AG, Base

$1018-2438 / 16 / 1683-0150 \$ 39.50 / 0$

E-Mail karger@karger.com

www.karger.com/iaa
Correspondence to: Assoc. Prof. Dr. Alain Jacquet

Department of Medicine, Faculty of Medicine, Chulalongkorn University 1873 Rama IV Road, Pathumwan

Bangkok 10330 (Thailand)

E-Mail alain.j@ chula.ac.th 
tant levels of Der $\mathrm{p} 23$ sensitizations in Thailand. Our study clearly suggested that rDer $\mathrm{p} 23$ is likely more appropriate for HDM allergy component-resolved diagnosis than HDM extracts.

(c) 2016 S. Karger AG, Basel

\section{Introduction}

The house dust mite (HDM) Dermatophagoides pteronyssinus is recognized as one of the most common sources of indoor airborne allergens triggering allergic sensitizations in approximately $20 \%$ of the population from industrialized countries $[1,2]$. Up to now, 17 different $D$. pteronyssinus allergen groups have been referenced and classified as major, intermediate or minor allergens [3]. Der $\mathrm{p}$ 1, a cysteine protease, and Der $\mathrm{p}$ 2, a LPS-binding protein, are considered as the most potent HDM allergens. Indeed, these two proteins are not only abundant in the bodies and feces of mites [4] but their IgE reactivities can reach up to $80-90 \%$ in HDM-allergic patients $[5,6]$. A new 8-kDa HDM allergen from fecal pellets, namely Der p 23, was recently classified as a major allergen. Indeed, in a European study, $74 \%$ of the HDM-allergic patients tested developed Der p 23 sensitization [7]. Such high IgE-binding frequency was unexpected because natural Der p 23 (nDer p 23) has been shown to be present only in tiny amounts in fecal pellets and in house dust and also poorly extracted under aqueous conditions. This limited nDer $\mathrm{p} 23$ release could likely be explained by the putative association of the allergen to the peritrophic matrix of mite feces, a semipermeable structure rich in proteins and chitin, which can protect the mite mid-gut from damage by food particles during digestion [8]. The difficulty of sourcing nDer $\mathrm{p} 23$ could be circumvented through heterologous expression of recombinant Der $\mathrm{p} 23$ (rDer p 23) which could provide virtually unlimited amounts of this allergen. Although the characterization of Der p 23 was mainly performed based on rDer $\mathrm{p} 23$ produced in Escherichia coli [7], a detailed analysis of the Der p 23 amino acid sequence suggested that this protein could contain post-translational modifications via the presence of (1) a $\mathrm{N}$-terminal leader sequence, (2) two regions rich in threonine residues prone to $\mathrm{O}$-glycosylation and (3) a putative C-terminal chitin-binding domain containing 4 cysteine residues involved in 2 disulfide bonds. Such modifications could be important for both the folding as well as the biological and allergenic properties of Der $\mathrm{p} 23$.

We report here the expression of rDer p 23 in Pichia pastoris. Contrary to E. coli, this yeast strain contains chi- tin and can express and secrete O-glycosylated proteins with appropriate disulfides. Following expression and purification, the prevalence of IgE reactivity to rDer $\mathrm{p} 23$ has, for the first time, been examined in a cohort of HDMallergic subjects from Thailand.

\section{Materials and Methods}

\section{Cloning of rDer $p 23$ in P. pastoris}

The mature Der p 23 coding gene sequence (207 bps corresponding to aa 22-90, with the numbering based on L7N6F8 Uniprot sequence) was amplified from total cDNA of D. pteronyssinus by PCR. Primers used were designed to incorporate XhoI and NotI enzyme restriction sites (underlined) at the $5^{\prime}$ - and $3^{\prime}$-end of the amplicon, respectively; forward primer: $5^{\prime}$-GGCTCGAGAAAAGAGAGGCTGAAGCTGCCAATGATAATGATGATGATCCTACCAC-3'; reverse primer: 5'-CCGCGGCCGCTTAAGTGCATGTTTCTTCATCTTC-3'. The purified PCR product was subsequently double-digested with XhoI and NotI and cloned into the yeast expression vector pPICZaA (Invitrogen) restricted by the same enzymes. The $P$. pastoris KM71 strain was subsequently transformed with the recombinant plasmid, pPICZaA-Der p 23, previously linearized with $B g l \mathrm{II}$, by electroporation using the Gene Pulser Xcell ${ }^{\mathrm{TM}}$ (Bio-Rad) at 1,500 V, $200 \Omega$ in 1 -mm electrode cuvette. The transformants were spread on YPDS agar plate ( $1 \%$ yeast extract, $2 \%$ peptone, $2 \%$ dextrose, $1 \mathrm{M}$ sorbitol and $2 \%$ agar) containing $100 \mu \mathrm{g} / \mathrm{ml}$ Zeocin (Invitrogen) and incubated at $30^{\circ} \mathrm{C}$ for 2-3 days.

Expression and Purification of rDer $p 23$

A colony PCR-positive clone was selected to inoculate an overnight culture in BMGY broth at $30^{\circ} \mathrm{C}$ under shaking conditions. The cells were subsequently pelleted and resuspended in BMMY containing $2 \%$ methanol to a final $\mathrm{OD}_{600 \mathrm{~nm}}$ of 5 to induce the protein expression. Methanol was added every $24 \mathrm{~h}$ to maintain the $2 \%$ concentration. For the purification of rDer $\mathrm{p} \mathrm{23,} \mathrm{the} \mathrm{culture}$ supernatant was collected by centrifugation after $48 \mathrm{~h}$ of induction and directly diluted 10 times with MilliQ water prior to $\mathrm{pH}$ adjustment to $\mathrm{pH}$ 3. The medium was subsequently applied to a S Sepharose XL column (GE Healthcare Life Sciences) equilibrated with $50 \mathrm{~mm}$ glycine- $\mathrm{HCl}(\mathrm{pH} 3)$. Elution was carried out in step-wise increases of $\mathrm{NaCl}$ concentration. The eluted fractions were immediately neutralized with $1 \mathrm{M}$ Tris- $\mathrm{HCl}$ ( $\mathrm{pH}$ 9). Fractions containing rDer p $23(200 \mathrm{mM} \mathrm{NaCl})$ were concentrated by ultrafiltration with a 3-kDa cut-off membrane (PALL) followed by gel filtration onto a Superdex 75 HR column 10/30 (Life Sciences) equilibrated with PBS ( $\mathrm{pH}$ 7.3). The fractions containing purified rDer $\mathrm{p} 23$ were pooled and stored at $-20^{\circ} \mathrm{C}$ for further analysis. Protein concentration was determined using a BCA protein assay kit (Pierce).

\section{Circular Dichroism}

Purified rDer p 23 was first dialyzed against $10 \mathrm{mM} \mathrm{NaH}_{2} \mathrm{PO}_{4}$ buffer ( $\mathrm{pH} 4.8$ ). Secondary structure composition of $0.2 \mathrm{mg} / \mathrm{ml}$ rDer p 23 was measured with Jasco J-815 circular dichroism (CD) spectrometer in a $0.1-\mathrm{cm}$-path-length quartz cuvette. CD spectra from 190-260 nm were recorded with $1 \mathrm{~nm}$ resolution at a speed of $50 \mathrm{~nm} / \mathrm{min}$ for 5 cycles. Data were converted to mean residue 
weight ellipticity $(\theta)_{\mathrm{mrw}}\left(\right.$ degrees $\cdot \mathrm{cm}^{2} \cdot \mathrm{dmol}^{-1}$ ) and then analyzed with CDPro software.

\section{Mite Feces Extraction and Immunoblot}

Purified feces (36 mg), separated by sequential sieving of $D$. pteronyssinus culture were extracted in $200 \mu \mathrm{l}$ of PBS $(137 \mathrm{mM}$ $\mathrm{NaCl}, 2.7 \mathrm{mM} \mathrm{KCl}, 10 \mathrm{mM}$ phosphate buffer, $\mathrm{pH} 7.3$ ) at $4{ }^{\circ} \mathrm{C}$ for $16 \mathrm{~h}$ under shaking conditions. Soluble extract was collected by centrifugation at $13,000 \mathrm{rpm}$. nDer p 23 was detected in the fecal extracts by Western blot following 15\% SDS-PAGE and protein transfer onto a nitrocellulose membrane (Bio-Rad). The membrane was then blocked with PBS, $1 \%$ (w/v) BSA and $0.05 \%(\mathrm{v} / \mathrm{v})$ Tween 20 at $4{ }^{\circ} \mathrm{C}$ for $16 \mathrm{~h}$. Next, the membrane was incubated with mouse anti-rDerp 23 polyclonal serum (the serum was produced through animal immunizations with purified rDer p 23 formulated with Alum adjuvant; data not shown) at a 1/2,000 dilution. As a control, the membrane was also incubated with preimmune serum. The membrane was further incubated with goat antimouse antibodies conjugated with horseradish peroxidase (KPL) at 1:5,000 dilution. The immunoreactive bands were detected using the chemiluminescent horseradish peroxidase substrate (Millipore) and X-ray film exposure (Kodak).

\section{Nano-Electrospray Ionization Mass Spectrometry Analysis: \\ Direct Infusion}

Prior to the nano-electrospray ionization mass spectrometry (nanoESI-MS) analysis, $20 \mu \mathrm{g}$ of rDer p 23 was desalted with a $\mathrm{C}_{4}$ ZipTip (Merck Millipore) and was eluted in $50 \mu \mathrm{l}$ of $50 \%$ acetonitrile $/ 0.1 \%$ formic acid. Next, the sample was infused at $1 \mu \mathrm{l} / \mathrm{min}$ into an Impact HD mass spectrometer equipped with a CaptiveSpray source (Bruker Daltonics). Acquisitions were performed in positive mode with the end-plate offset and capillary voltages set at -500 and $1,200 \mathrm{~V}$, respectively. The drying gas flow rate and dry heater were set at 4.0 liters $/ \mathrm{min}$ and $180^{\circ} \mathrm{C}$, respectively. MS spectra were acquired over the $\mathrm{m} / \mathrm{z}$ range of $50-3,000$ with a scan rate of $1 \mathrm{~Hz}$. MS calibration was performed using the internal lockmass at $\mathrm{m} / \mathrm{z} 1221.9906$ (Agilent). Charge state deconvolution was performed using the maximum entropy algorithm.

Chitin-Binding Assay

Chitin beads (New England Biolabs) and shrimp chitin (Sigma) were used in the affinity binding assay. The insoluble chitin (50 $\mu$ of chitin bead suspension or $1 \mathrm{mg}$ of shrimp chitin) was equilibrated in PBS and incubated with $20 \mu \mathrm{g}$ rDer p 23, rDer p 5 (as a negative control), wheat germ agglutinin (WGA, as a positive control, Vector) or $100 \mu \mathrm{l}$ of HDM fecal extracts at room temperature for $60 \mathrm{~min}$ with orbital shaking. Supernatants were collected by centrifugation at 13,000 rpm for $5 \mathrm{~min}$ and the chitin pellets were washed 5 times with PBS. Bound proteins were then eluted by incubating the chitin pellet in Laemmli buffer at $95^{\circ} \mathrm{C}$ for $5 \mathrm{~min}$. Both supernatant and eluted fractions were analyzed on $15 \%$ SDS-PAGE and Coomassie blue staining, whereas the HDM fecal extracts were detected by immunoblot with specific rDer $\mathrm{p}$ 23 antibodies.

\section{Alpha Mannosidase Digestion}

Purified rDer p 23 was digested with Jack bean $\alpha$-mannosidase (Sigma-Aldrich) at a 1:20 enzyme/substrate ratio in a $\mathrm{pH} 4$ buffer at $37^{\circ} \mathrm{C}$ for $16 \mathrm{~h}$. The solution was neutralized with $1 \mathrm{M}$ Tris- $\mathrm{HCl}$ $\mathrm{pH} 9$ and ultrafiltrated through a $30-\mathrm{kDa}$ cut-off membrane
(Vivaspin, GE Healthcare) to remove mannosidase. The flowthrough containing rDer p 23 was subsequently concentrated through a $3-\mathrm{kDa}$ cut-off membrane (Vivaspin, GE Healthcare). The filtrate was collected and the protein concentration was determined using a BCA protein assay kit (Pierce).

\section{IgE Reactivity to rDer $p 23$}

Sera from 222 patients with HDM-associated allergic rhinitis or asthma were obtained from the King Chulalongkorn Memorial Hospital ( $\mathrm{n}=95)$, Children Hospital $(\mathrm{n}=81)$, Ramathibodi Hospital $(\mathrm{n}=32)$ and Phramongkutklao Hospital $(\mathrm{n}=14)$, respectively. The $D$. pteronyssinus-specific IgE antibodies (0.36-100 $\mathrm{kU}_{\mathrm{A}} / \mathrm{l}$ ) were assayed with the ImmunoCAP System (Phadia). Each patient provided written informed consent. As negative controls, sera from non-allergic subjects were also collected $(<0.35 \mathrm{kU} / \mathrm{l}, \mathrm{n}=67)$. The study was approved by the Ethics Committees of the Faculty of Medicine, Chulalongkorn University (IRB 023/55), the Children Hospital (IRB 195/2556), the Faculty of Medicine, Ramathibodi Hospital, Mahidol University (IRB 0356-34) and the Phramongkutklao College of Medicine (IRB S039Q/57_EXP).

For the IgE-binding assays, ELISA microplates were coated with $500 \mathrm{ng} /$ well of rDer p 23 or deglycosylated rDer p 23 or rDer p 2 at $4^{\circ} \mathrm{C}$ for overnight. The plates were then washed with PBSTween 20, 0.05\% (PBS-T) and blocked with PBS-T containing 1\% BSA (PBS-T-BSA) for $1 \mathrm{~h}$ at $37^{\circ} \mathrm{C}$. Serum samples were diluted at $1 / 8$ in PBS-T-BSA and incubated at $37^{\circ} \mathrm{C}$ for $1 \mathrm{~h}$. The plates were then washed again with PBS-T and further incubated with a $1 / 5,000$ dilution of goat biotinylated anti-human IgE (KPL) at $37^{\circ} \mathrm{C}$ for $1 \mathrm{~h}$. Next, the plates were washed with PBS-T and incubated with a 1/2,000 dilution of streptavidin-peroxidase (BD Biosciences) for $1 \mathrm{~h}$ at room temperature. The allergen-antibody complex was detected with TMB substrate (BD Biosciences) and the reaction was stopped with $0.5 \mathrm{M}$ sulfuric acid. OD was determined at $450 \mathrm{~nm}$ using an iMark microplate reader (Bio-Rad). Patient serum was considered positive when the measured OD value was higher than the cut-off value, established as the mean OD values of negative control sera plus 2 standard deviations.

\section{Rat Basophil Degranulation Assay}

Genetically modified rat basophil leukemia (RBL) cell line expressing human FceRI receptor, RBL-SX38, were cultured in 96well culture plate at a cell density of $1.5 \times 10^{5}$ cells/well in RPMI 1640 medium supplemented with $10 \% \mathrm{FBS}$ at $37^{\circ} \mathrm{C}$ for $3 \mathrm{~h}$ [9]. Cells were subsequently primed for $16 \mathrm{~h}$ with human sera (positive or negative for rDer p 23 or rDer p 2-specific IgE) at a 1/10 dilution. Cells were then washed with PBS and incubated with phenol-free RPMI 1640 containing $1 \mathrm{mg} / \mathrm{ml}$ BSA for $15 \mathrm{~min}$ before the addition of serial rDer p 23 or rDer p 2 dilutions ( $0.00001 \mathrm{up}$ to $1 \mu \mathrm{g} / \mathrm{ml}$ ) for $30 \mathrm{~min}$. To evaluate the maximum degranulation, control cells were treated with $5 \%(\mathrm{v} / \mathrm{v})$ Triton X-100. The $\beta$-hexosaminidase release was measured following incubations of culture supernatant $(50 \mu \mathrm{l})$ into the same volume of prewarmed $2.5 \mathrm{mM}$ p-nitrophenyl $\mathrm{N}$-acetyl$\beta$-D-glucosaminide for $3 \mathrm{~h}$. Finally, the reaction was stopped with $150 \mu \mathrm{l}$ of $1 \mathrm{M}$ Tris- $\mathrm{HCl}$ ( $\mathrm{pH}$ 9) and $\mathrm{A}_{415} \mathrm{~nm}$ was subsequently read with an iMark microplate absorbance reader (Bio-Rad).

\section{Statistical Analysis}

Data were compared using Student's t test. $\mathrm{p}<0.05$ was considered to be significant. 
Fig. 1. Expression and purification of rDer p 23 from transformed KM71 P. pastoris. a SDS-PAGE analysis of rDer p 23 secretion profile under $2 \%$ methanol induction at $24,48,72,96,120$ and $144 \mathrm{~h}$. NI = Noninduced. b Purified rDer p 23 after ion exchange and gel filtration chromatography. $\mathrm{M}=$ Molecular weight markers.

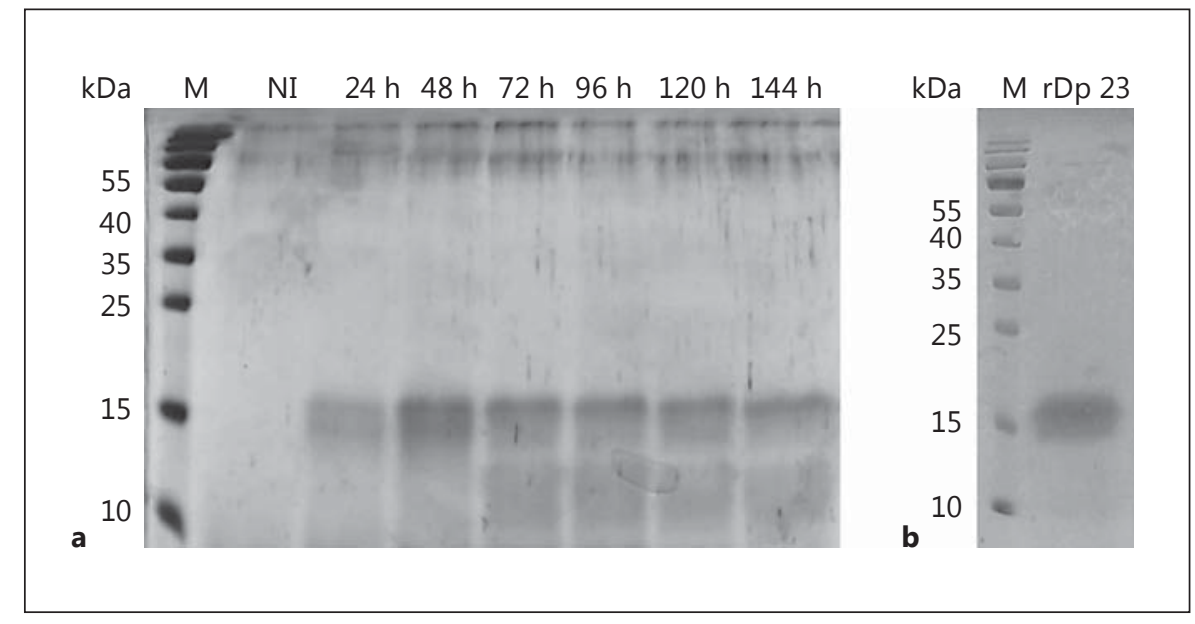

\section{Results}

\section{Cloning, Expression and Purification of Mature}

rDerp 23

Using 2 specific primers derived from the sequence EU414751.1 (GenBank accession No. [7]), we successfully amplified the cDNA encoding the mature form of Der p 23 (aa 22-90) from a D. pteronyssinus total cDNA preparation. The DNA sequence of the amplicon perfectly matched the one from the GenBank database. The mature Der p 23 coding sequence was subsequently cloned into $P$. pastoris $\mathrm{pPICZaA}$ expression vector, directly downstream to the yeast $\alpha$-mating factor leader sequence for the secretion of the allergen without any additional amino acids.

Following the transformation of the P. pastoris KM71 strain with the recombinant linearized plasmid, zeocinresistant colonies were screened for the presence of Der $p$ 23 cDNA by colony PCR. Positive colonies were cultured in shake flasks at $30^{\circ} \mathrm{C}$ and expression of rDer p 23 was triggered by the addition of methanol into the culture medium at a final concentration of $2 \%$. The methanol induction was shown to stimulate the production of secreted rDer $\mathrm{p} 23$, which migrated as a $15-\mathrm{kD}$ a protein on SDSPAGE (fig. 1a). The expression was shown to reach a maximum after $48 \mathrm{~h}$ of induction. For a longer expression period, Der $\mathrm{p} 23$ degradation products migrating at about $10 \mathrm{kDa}$ were observed (fig. 1a). MS analysis of SDS-PAGE bands confirmed that both the 10- and $15-\mathrm{kDa}$ bands contain rDer p 23-related sequences, although the mature Der p 23 theoretical molecular weight deduced from the amino acid sequence was $7.9 \mathrm{kDa}$. The $15-\mathrm{kDa}$ band corresponded with the intact mature rDer $\mathrm{p} 23$ bearing hex- ose residues (8-16 residues) with a mass ranging from 9-10.5 kDa, while the $10-\mathrm{kDa}$ band corresponded with truncated rDer $\mathrm{p} 23$ with no hexose and with a mass of approximately $6.5 \mathrm{kDa}$ (data not shown). rDer p 23 was purified to homogeneity from the culture supernatants by a combination of cation exchange and size-exclusion chromatographies (fig. 1b). The SDS-PAGE profile of purified rDer $\mathrm{p} 23$ did not show the presence of a dimeric form as previously reported by Weghofer et al. [7] for rDer $\mathrm{p} 23$ produced in bacteria.

Der p 23 Produced in P. pastoris Is an O-Glycoprotein

The nanoESI-MS characterization of purified rDer $\mathrm{p}$ 23 demonstrated the presence of N-terminal sequence heterogeneity, but no host cell proteins were detected. Together with a large percentage of intact, full-length, mature rDer p 23 (aa 22-90, 77\%), 4 truncated forms of rDer p 23 were detected, i.e. A (aa 33-90, 5\%), B (aa 3690, 4\%), C (aa 37-90, 10\%) and D (aa 39-90, 3\%; fig. 2a). Noteworthy, no mass increment associated with hexose residues $(162.05 \mathrm{Da})$ was observed on these fragments. Only the intact form of rDer p 23 carried such oligosaccharide structures, suggesting that threonine residues at positions 30-32 could represent an O-glycosylation site (fig. 2a). Quadrupole-time-of-flight MS investigations also established that rDer $\mathrm{p} 23$ has 2 intra-disulfide bonds through the determination of the protein experimental mass $(7,972.37 \mathrm{Da}$; fig. $2 \mathrm{a})$, which fitted with the nonreduced theoretical monoisotopic mass of Der p 23 (aa 2290, 7,972.37 Da).

As proteins produced in yeast are commonly O-glycosylated through mannose residues [10], we then treated rDer p 23 with Jack bean a-mannosidase which 


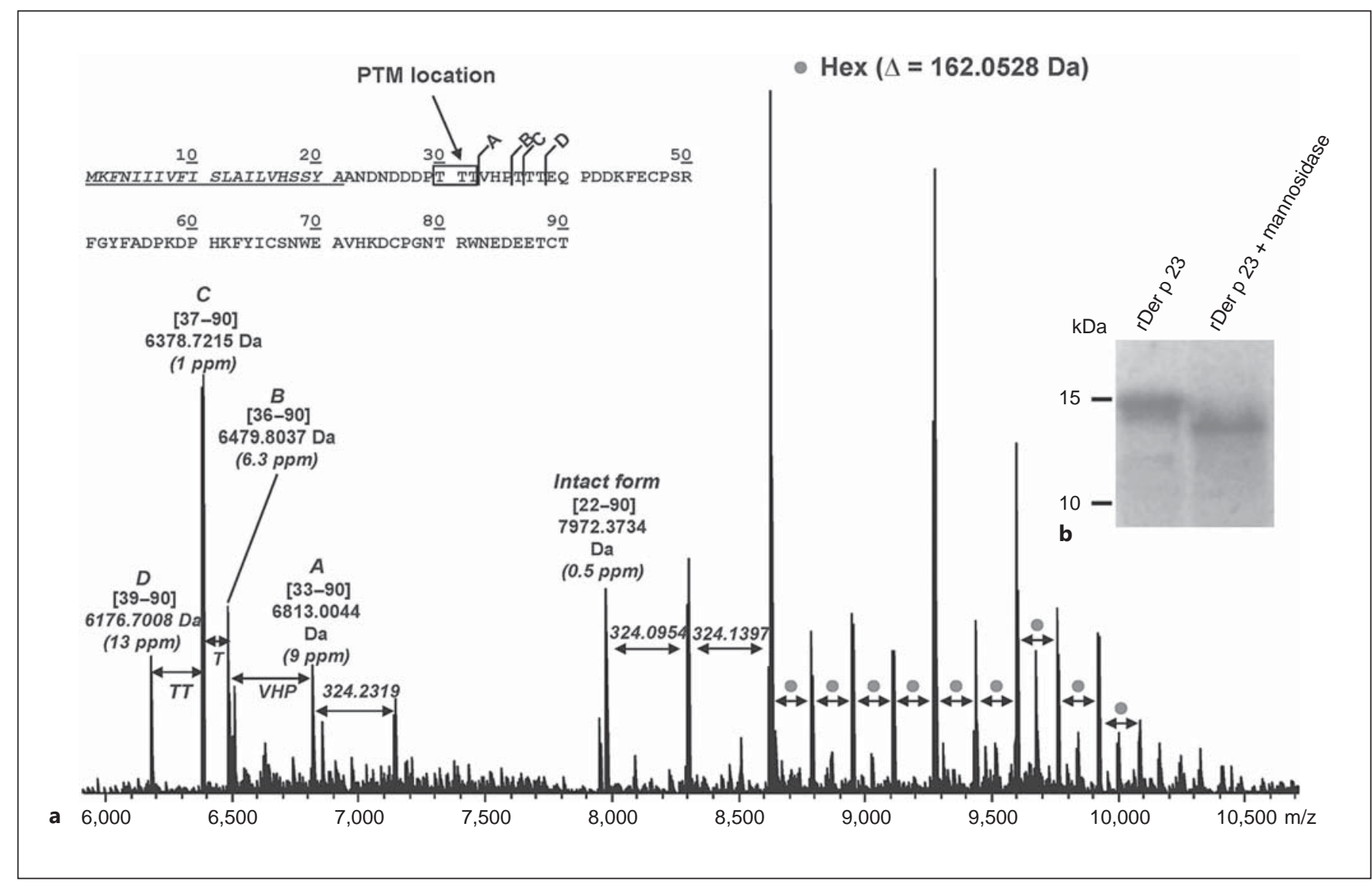

Fig. 2. NanoESI-MS analysis and deglycosylation of rDer p 23. a Deconvoluted mass spectrum of purified rDer $\mathrm{p}$ 23. The fulllength amino acid sequence of Der $\mathrm{p} 23$ is shown on the top left corner. Amino acid sequence 1-21 (underlined) is the signal peptide while mature intact form of rDer p 23 consists in stretch 2290. Four truncated forms of rDer p 23 are labeled: A (aa 33-90),
B (aa 36-90), C (aa 37-90) and D (aa 39-90). Hexose residues are represented with dots. b Purified rDer p 23 was incubated with $\alpha$-mannosidase overnight at $37^{\circ} \mathrm{C}$ and analyzed by SDS-PAGE. Purified rDer p 23 was also incubated at the same condition as controls. PTM = Post-translational modification.

\section{Detection of nDer p 23 in HDM Feces Extracts}

Through chemiluminescence detection, the specific antibodies raised to rDer $\mathrm{p} 23$ were also able to detect nDer $\mathrm{p} 23$ from aqueous extracts of purified HDM fecal pellet preparations (fig. 4). Our results suggest that the recombinant and natural forms of Der p 23 share common B cell epitopes.

\section{IgE Reactivity to rDerp 23}

The IgE-binding frequency to rDer p 23 was evaluated in a cohort of Thai HDM-allergic patients $(n=222)$ by indirect ELISA. All the selected sera displayed an ImmunoCap $D$. pteronyssinus-specific IgE value $>0.35$ $\mathrm{kU}_{\mathrm{A}} / \mathrm{l}$. Of the HDM-positive sera $(\mathrm{n}=119), 54 \%$ showed specific IgE reactivity to $\mathrm{rDer}$ p 23 whereas IgE reactiv- 


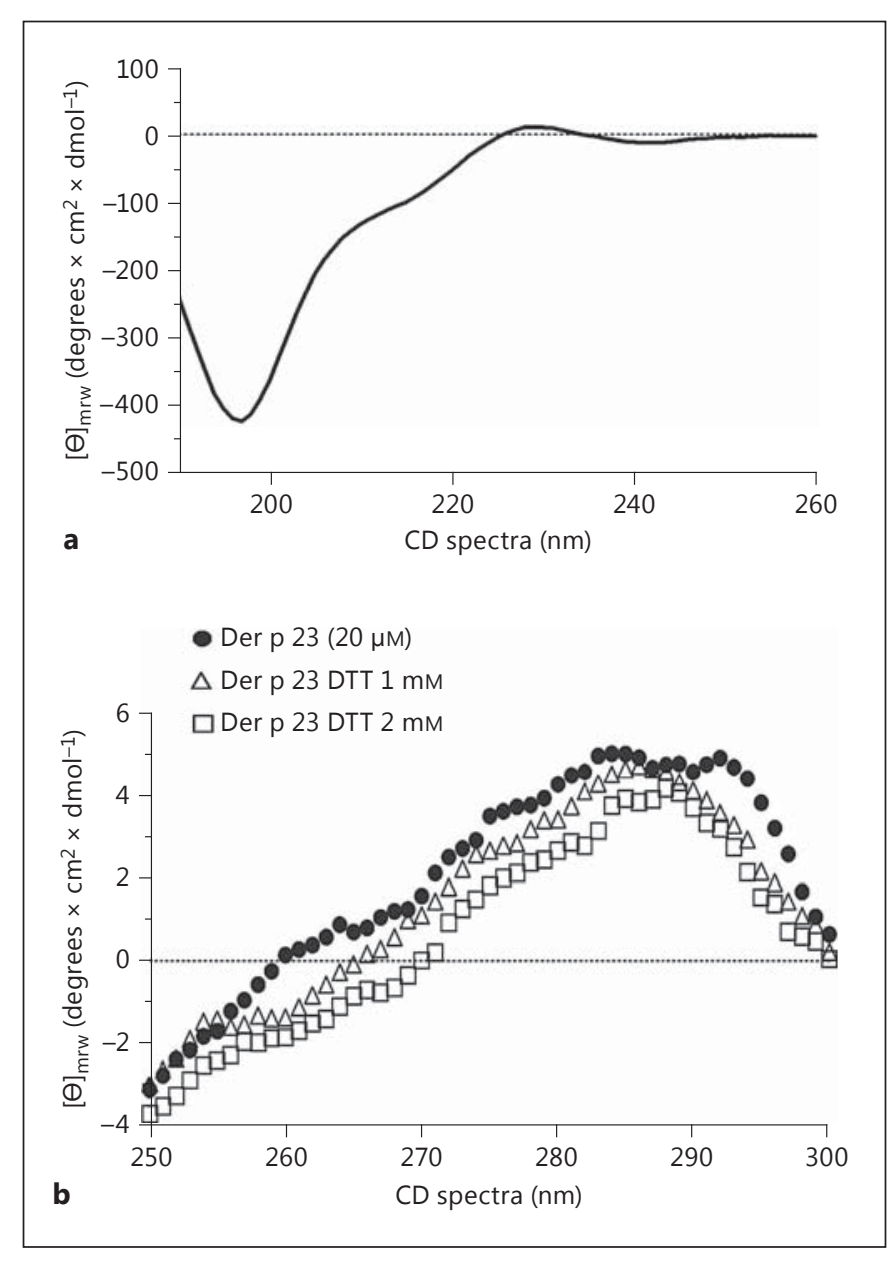

Fig. 3. UV-circular dichroism analysis of purified rDer p 23. a CD spectra of untreated purified rDer p 23 at $25^{\circ} \mathrm{C}$. b rDer p 23 was treated with 1 or $2 \mathrm{mM} \mathrm{DTT}$. All the analyses were done using a J-815 CD spectrophotometer.

ity to rDer p 2 was detected in $67 \%$ of the same miteallergic individuals $(n=148)$, confirming that Der $\mathrm{p} 23$ is a major allergen. A significant positive correlation between OD values to Der p 23 and to Der p 2 was demonstrated, even though several patients were monosensitized to one of these two allergens (Pearson's correlation; $\mathrm{p}<0.0001$; data not shown). We further evaluated the influence of rDer p 23 oligosaccharides on its IgEbinding properties. By comparing the OD values of Der p 23-positive sera obtained from the ELISA against rDer p 23 with deglycosylated rDer p 23, we confirmed that the glycan structure of rDer $\mathrm{p} 23$ did not impact the IgE binding (online suppl. table 1 ; for all online suppl. material, see www.karger.com/doi/10.1159/000442176; $\mathrm{p}=$ $0.8762)$.

IgE and Chitin-Binding Activities of Glycosylated Der p 23

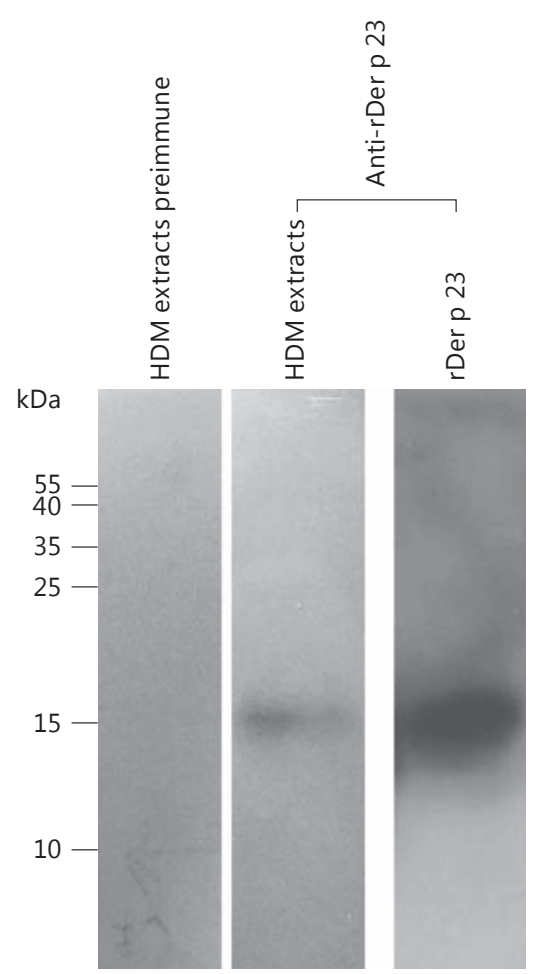

Fig. 4. Detection of Der $\mathrm{p} 23$ in HDM feces from D. pteronyssinus. Nitrocellulose-blotted feces extracts were incubated with mouse polyclonal antibody against rDer $\mathrm{p} 23$ and preimmune serum of a Der p23-immunized mouse. Bound antibodies were detected with rabbit anti-mouse-HRP and the luminescence signal was captured by radiography film.

\section{Allergenicity of rDer $p 23$}

To demonstrate that rDer p 23 can trigger basophil degranulation through interactions with FceRI-bound IgE, a basophil degranulation assay was performed using RBL-SX38 cells. The allergenic activity of rDer $\mathrm{p} 23$ was demonstrated using 3 representative sera from HDM-allergic patients that contained Der p 23-specific IgE and comparing these with rDer $\mathrm{p} 2$ allergenic activity (fig. 5). rDer p 23 was able to induce basophil degranulation at concentrations ranging from 0.0001 to $1 \mu \mathrm{g} / \mathrm{ml}$. Such allergenic activity was comparable to that displayed by rDer p 2. No significant degranulation was observed with buffer only, rDer p 23 only or with serum that was non-HDMallergic. 

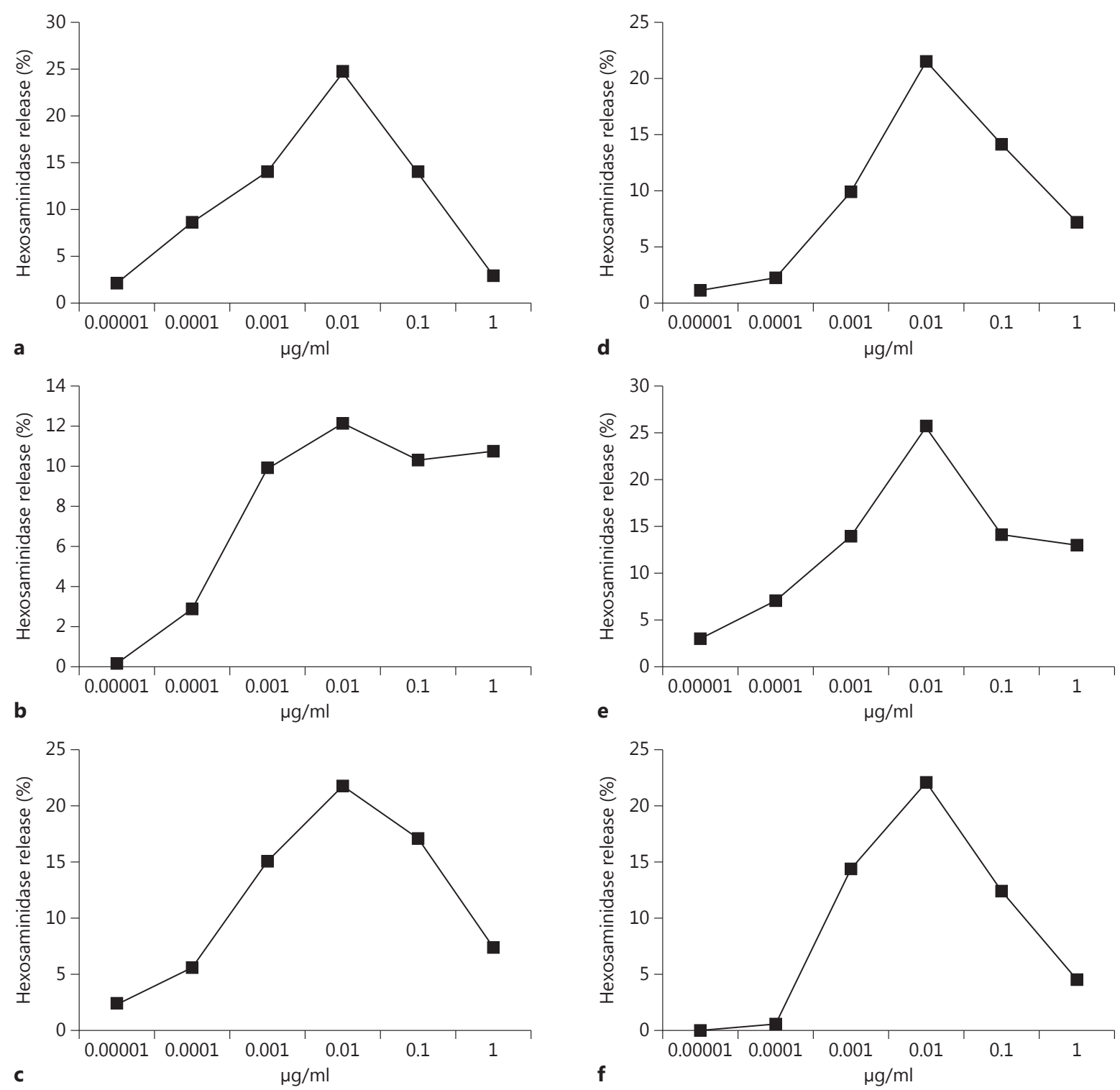

Fig. 5. RBL-SX38 degranulation by rDer $\mathrm{p} 23$ and rDer $\mathrm{p} 2$. The cells were primed for $16 \mathrm{~h}$ with sera from $3 \mathrm{HDM}$-allergic patients, containing rDer p 23- (a-c) or rDer p 2-specific IgE (d-f) and subsequently stimulated with serial dilution of purified rDer p 23

or $\mathrm{rDer}$ p 2 for $30 \mathrm{~min}$. Degranulation was measured via $\beta$ hexosaminidase activity. Percentage of degranulation was presented as a subtraction of spontaneous released over total lysis with Triton X-100.

rDer p 23 Does Not Display Chitin-Binding Activity

We evaluated the chitin-binding activity of rDer $\mathrm{p} 23$ using two different sources of insoluble chitins: chitin beads and shrimp-shell chitin. Under our experimental conditions, rDer $\mathrm{p} 23$ as well as nDer $\mathrm{p} 23$ from fecal pellet extracts could not bind to the aforementioned forms of chitin resins (fig. 6). By contrast, the positive control, wheat germ agglutinin (WGA), a well-known chitinbinding protein, bound efficiently to the two chitin-based matrices. On the other hand, rDer p 5, which does not display sequence homology to the chitin-binding domain, was used as negative control. To confirm that putative traces of sugars in the Der p 23 preparation could impair the protein binding to chitin by competition, we 


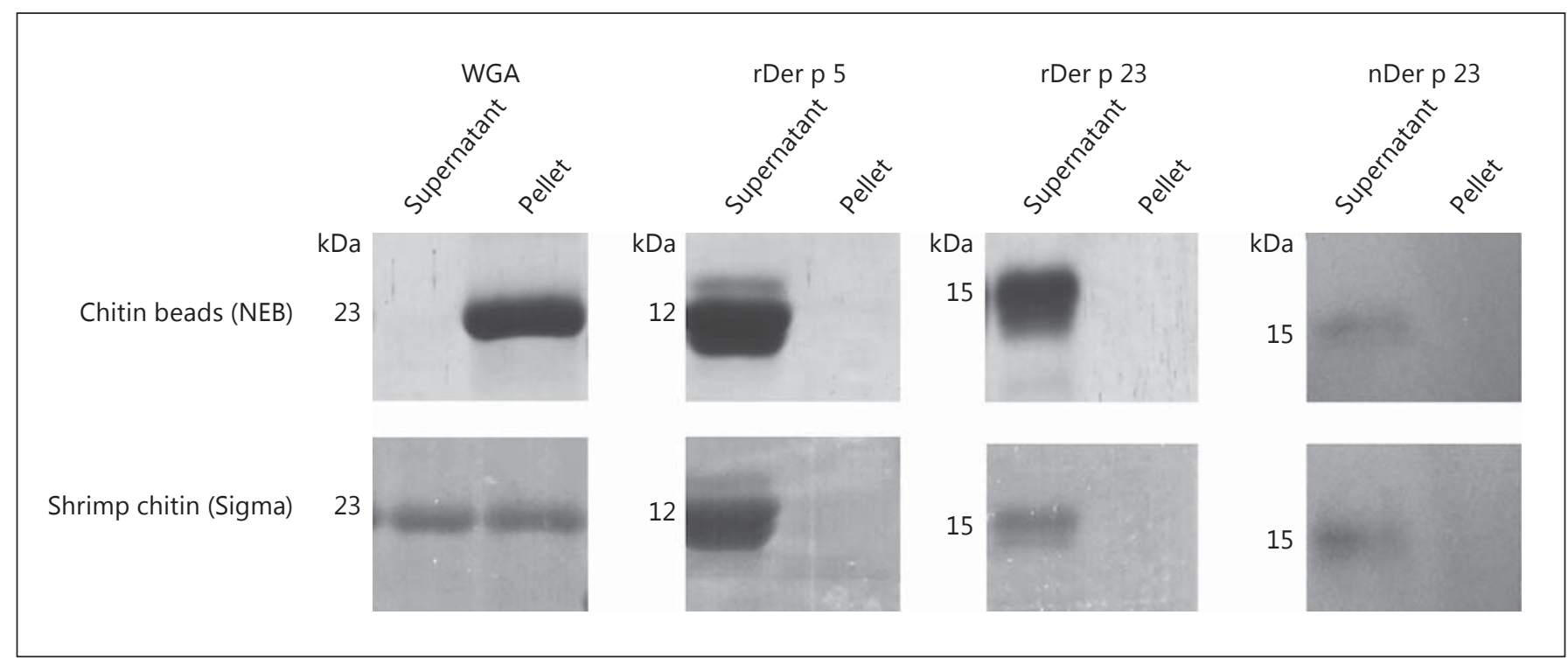

Fig. 6. Chitin-binding assay. Chitin-binding activity of rDer 23 produced in $P$. pastoris was assessed by incubating the protein with insoluble chitin bead and shrimp chitin. The fractions were then analyzed on SDS-PAGE, except nDer $\mathrm{p} 23$ was analyzed by immunoblot. WGA was used as a positive control and rDer p5 as a negative control.

demonstrated that WGA can still bind chitin in the presence of $P$. pastoris culture medium (data not shown). It must also be pointed out that a GST-Der $\mathrm{p} 23$ fusion protein produced in bacteria was also not able to bind to chitin under our experimental conditions (data not shown). Finally, to exclude that the absence of binding was not related to differences in chitin structures between mite feces and chitin beads or shrimp chitin, we performed the same assay using a suspension of HDM fecal pellets. The staining of fecal pellets with calcofluor demonstrated clearly the presence of the peritrophic matrix made in the chitin structures (online suppl. fig. 1). Once again, rDer $\mathrm{p}$ 23 did not interact with HDM fecal pellets under our experimental conditions where WGA could be associated with these particles (online suppl. fig. 1). Altogether, our data suggest that Der p 23 alone does not display any chitin-binding activity.

\section{Discussion}

A recent paper provided evidence of Der $\mathrm{p} 23$ as a new major $D$. pteronyssinus allergen. The IgE reactivity to a recombinant form of Der p 23 produced in E. coli was comparable to those raised to Der p 1 and Der p 2 (74\% in European cohorts of HDM-allergic patients) [7]. This high IgE-specific frequency is somewhat challenging. Indeed, Der p 23 has been shown as mainly an unfolded protein, present in very tiny amounts in house dust. Moreover, its aqueous extraction yield from fecal pellets is very low, probably because it is found next to the peritrophic matrix surrounding the feces. Such semipermeable matrix, produced in the mite mid-gut during food digestion to prevent the epithelium from damage by abrasive food particles and pathogens, is mainly composed of proteins and chitin fibrils [12]. According to the capacity of chitin to trigger innate immunity to shape a Th2-biased cytokine environment, the potent allergenicity of Der $p$ 23 could result from its association with chitin [13, 14]. Although the Der p 23 C-terminal region displays homologies with a chitin-binding domain type 2 , the interactions between the allergen and chitin structure have still to be shown.

In this study, we selected the P. pastoris expression system to produce and characterize a recombinant mature form of Der $\mathrm{p}$ 23. The rationale for this selection was based on the presence in the Der p 23 primary structure of a leader peptide for secretion, 2 threonine stretches which could represent $\mathrm{O}$-glycosylation sites and 4 cysteine residues in the putative $\mathrm{C}$-terminal chitin-binding domains which could be involved in 2 disulfides. All 3 of these post-translational modifications, which could be 
critical for the allergenic properties and stability of Der $p$ 23 , can be performed by yeast. It must be pointed out that HDM allergens could be naturally glycosylated because glycan structures such as mannosylations were detected in natural Der p 1; such modifications could not take place when proteins are produced in bacteria [15]. Fulllength mature and $\mathrm{O}$-mannosylated rDer $\mathrm{p} 23$ was successfully secreted by $P$. pastoris. The purified protein migrated as a $15-\mathrm{kDa}$ band onto SDS-PAGE, whereas the calculated mass by MS analysis was $10.1 \mathrm{kDa}$ (full-length, longest mannose chains). This abnormal SDS-PAGE migration could likely result from the proline content in Der p 23 because proline-rich proteins commonly display a decreased electrophoretic mobility [16]. Under our experimental conditions, $\mathrm{N}$-terminal truncations of aa 1117 were observed, particularly when the methanol induction exceeded $48 \mathrm{~h}$. We speculate that these truncations/ degradations could already have occurred via the yeast secretory pathway because stability studies clearly indicated that purified rDer $\mathrm{p} 23$ is highly stable, even when the protein is stored at $37^{\circ} \mathrm{C}$ for 14 days (data not shown). It must be pointed out that MS analysis of aqueous extracts from fecal pellets also demonstrated the sensitivity of Der p 23 to proteolysis because, although peptide fragments can be clearly identified, the detection of the fulllength natural allergen was difficult to achieve (data not shown). The secondary structure analysis by CD confirmed that, similarly to the recombinant form produced in bacteria, Der p 23 expressed in yeast is mainly folded, with a random coil structure and disulfides present, suggesting that Der $\mathrm{p} 23$ likely displays more linear than conformational antibody-binding epitopes on the allergen surface [17]. This is in agreement with the capacity of purified specific polyclonal antibodies to detect rDer $\mathrm{p} 23$ under native (ELISA) or denaturing (immunoblot after SDS-PAGE) conditions.

Using a cohort of sera from 222 Thai HDM-allergic patients, we confirmed that Der p 23 is an important allergen because the frequency of Der p 23-specific IgE reached $54 \%$. Noteworthy is that IgE reactivity was lower than that measured for the typical major allergen Der $\mathrm{p} 2$ (67\%) using the same sera. To our knowledge, this is the first time that the IgE-binding frequencies of Der $\mathrm{p} 2$ and Der p 23 have been determined in atopic patients from Thailand. However, RBL degranulation assays produced clear evidence of the potent allergenic activity of Der $\mathrm{p} 23$. Higher IgE reactivity to Der p $23(74 \%, \mathrm{n}=347)$, using a recombinant form produced in $E$. coli, was observed in European cohorts of HDM-allergic patients [7]. It must be pointed out that, based on seroepidemiological data from 1,300 HDM-allergic patients from Canada, Europe, Japan and the USA, around $80 \%$ of these patients had IgE reactivity to Der p 2 but around $47 \%$ reacted to Der p 23 (our currently unpubl. data). Thus, we believe that the IgE-binding frequencies found within the Thai population for Der p 23 (54\%) and Der p 2 (67\%) are comparable those observed in other populations. The lower prevalence of Der p 23 sensitivity in Thai HDM-allergic patients cannot be explained by the O-mannosylations of the $\mathrm{N}$-terminal domain of yeast-expressed allergen, as we demonstrated that the glycan structure of rDer p 23 did not influence IgE binding. Moreover, it was recently demonstrated that major IgE epitopes of Der $\mathrm{p} 23$ are present at the C-terminal part corresponding to the putative chitin-binding domain [17]. There is increasing evidence that the allergenicity of HDM allergens can be mediated by their interactions with microbial compounds which are able to activate innate immune signaling pathways, leading to the induction of Th2-biased allergen-specific adaptive responses [18]. In this context, the allergenic activity of Der $\mathrm{p} 23$ could result from association with chitin structures, according to the presence of the putative chitin-binding domain at the C-terminus of the protein. Using two different sources of chitin, our results suggest that rDer p 23 and nDer $\mathrm{p} 23$ are not capable of binding to this sugar polymer. Similar results were obtained with a recombinant GST-Der $\mathrm{p} 23$ fusion protein produced in bacteria (data not shown), suggesting that $\mathrm{O}$-glycosylation could not interfere with any chitin binding. The putative chitin-binding domain of Der p 23 contains only 4 cysteine residues whereas the canonical typical peritrophin- $A$ and chitin-binding 2 domains contain $\geq 6$ conserved cysteines. Moreover, chitin-binding activity has been commonly demonstrated in proteins containing at least 2 of these domains $[19,20]$. Some proteins could display chitin-binding activity through a domain containing only 5 cysteine residues as Blo t 12 [21]. To our knowledge, the interaction between chitin and proteins harboring a domain with only 4 cysteines was not demonstrated up to now. As we also demonstrated that rDer p 23 could not bind to chitin structures present on the surface of mite fecal pellets, we speculate that the localization of Der $\mathrm{p} 23$ into the peritrophic matrix of the mite could result from interactions with molecular partners other than chitin, or from the existence of oligomeric forms of the allergen exposing the appropriate 4 -cysteine-based chitin-binding domains to interact with chitin. It must also be pointed out that the Der f 23 amino acid sequence was shown to contain a putative chitin binding domain with 5 cysteine residues; $4 / 5$ cysteine residues are conserved in Der p 23 
[22]. However, the chitin-binding properties of Der $\mathrm{f} 23$ have yet to be elucidated.

The role of glycosylation in the recognition and uptake of allergens by the innate immune system through C-type lectin receptors was also shown to be important for the initiation of the allergic response [23]. In this study, we observed an O-glycosylation site made of a stretch of threonine residues in the $\mathrm{N}$-terminus of Der $\mathrm{p}$ 23. Although the presence of O-glycans has still to be demonstrated in natural Der p 23, O-glycosylation of HDM allergens can be performed in mites, as previously shown for the HDM chitinase Der $f 15$ [24]. Moreover, on Western blot, nDer p 23 and rDer p 23 had the same molecular weight, indicating that nDer $\mathrm{p} 23$ could also be modified.

In conclusion, the HDM major allergen rDer $\mathrm{p} 23$ displays O-glycan-independent IgE reactivities but no chitin-binding activity. Our findings confirm that Der p 23 displays potent allergenic activity, despite the fact that it is a protease-sensitive and poorly extractable allergen from mite fecal pellets. Recombinant forms of Der $\mathrm{p} 23$ should be included in the panel of allergens for the component-resolved diagnosis of HDM allergy as well as in the design of specific immunotherapeutic treatments. Whereas rDer p 23 produced in E. coli and P. pastoris could be interchangeable for the in vitro detection of Der p 23 sensitizations, we believe that allergen production in yeast, due to the absence of contaminating endotoxins, would be more convenient for allergen-specific immunotherapeutic treatments or allergy diagnosis by skin-prick testing. Further studies are needed to evaluate the release of Der p 23 from the fecal pellets at the level of the airway epithelium and, notably, the contribution of the HDM chitinases Der p 15 and Der $p 18$ in the process. The elucidation of the Der $\mathrm{p} 23$-induced innate immune signaling pathways would shed a new light on the allergenic determinant(s) of Der p 23.

\section{Appendix}

The Mite Allergy Research Cohort (MARC) Study Team consisted of Pattarawat Thantiworasit and Pinya Pulsawat at the King Chulalongkorn Memorial Hospital, Tassalalpa Daengsuwan at the Children Hospital, Wannada Laisuan, Malinee Tongdee, Nizchapha Dchapaphapeaktak at the Ramathibodi Hospital and Tadech Boonpiyathad at the Phramongkutklao College of Medicine.

\section{Acknowledgements}

We warmly thank the MARC Study Team for the sera as well as clinical data collections. Wai Tuck Soh was supported under the Scholarship Program for ASEAN countries 2013 by Chulalongkorn University, Bangkok, Thailand. This work was partly funded by the 90th Anniversary of Chulalongkorn University Scholarship under the Ratchadaphisek Somphot Fund, RSA56 Thailand Research Fund and the National Research University Project Office of Higher Education Commission (WCU003-HR57). Kiat Ruxrungtham is supported by the Senior Research Scholar of the Thailand Research Fund, and the Research Professor Program of Chulalongkorn University, Bangkok, Thailand.

\section{Disclosure Statement}

The authors declare that there are no conflicts of interests.

\section{References}

1 Zock J-P, Heinrich J, Jarvis D, Verlato G, Norbäck D, Plana E, et al: Distribution and determinants of house dust mite allergens in Europe: the European Community Respiratory Health Survey II. J Allergy Clin Immunol 2006;118:682-690.

2 Gregory LG, Lloyd CM: Orchestrating house dust mite-associated allergy in the lung. Trends Immunol 2011;32:402-411.

3 Raulf M, Bergmann KC, Kull S, Sander I, Hilger $\mathrm{C}$, Brüning T, et al: Mites and other indoor allergens - from exposure to sensitization and treatment. Allergol J Int 2015;24:68-80.

4 Moingeon P: Progress in the development of specific immunotherapies for house dust mite allergies. Expert Rev Vaccines 2014;13:14631473 .

IgE and Chitin-Binding Activities of Glycosylated Der p 23
5 Bronnert M, Mancini J, Birnbaum J, Agabriel C, Liabeuf V, Porri F, et al: Component-resolved diagnosis with commercially available D. pteronyssinus Der $\mathrm{p} 1$, Der $\mathrm{p} 2$ and Der $\mathrm{p}$ 10: relevant markers for house dust mite allergy. Clin Exp Allergy 2012;42:1406-1415.

6 Weghofer M, Thomas WR, Kronqvist M, Mari A, Purohit A, Pauli G, et al: Variability of $\mathrm{IgE}$ reactivity profiles among European mite allergic patients. Eur J Clin Invest 2008; 38:959-965.

7 Weghofer M, Grote M, Resch Y, Casset A, Kneidinger M, Kopec J, et al: Identification of Der p 23, a peritrophin-like protein, as a new major Dermatophagoides pteronyssinus allergen associated with the peritrophic matrix of mite fecal pellets. J Immunol 2013;190:30593067.
8 Hegedus D, Erlandson M, Gillott C, Toprak $\mathrm{U}$ : New insights into peritrophic matrix synthesis, architecture, and function. Annu Rev Entomol 2009;54:285-302.

9 Wiegand TW, Williams PB, Dreskin SC, Jouvin $\mathrm{MH}$, Kinet JP, Tasset D: High-affinity oligonucleotide ligands to human IgE inhibit binding to Fc epsilon receptor I. J Immunol 1996;157:221-230.

10 Willer T: O-mannosyl glycans: from yeast to novel associations with human disease. Curr Opin Struct Biol 2003;13:621-630.

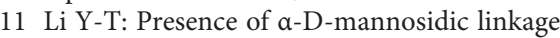
in glycoproteins. J Biol Chem 1966;241:10101012.

12 Tellam RL, Wijffels G, Willadsen P: Peritrophic matrix proteins. Insect Biochem Mol Biol 1999;29:87-101. 
13 Roy RM, Wüthrich M, Klein BS: Chitin elicits CCL2 from airway epithelial cells and induces CCR2-dependent innate allergic inflammation in the lung. J Immunol 2012;189:25452552.

14 Reese TA, Liang H-E, Tager AM, Luster AD, Van Rooijen N, Voehringer D, et al: Chitin induces accumulation in tissue of innate immune cells associated with allergy. Nature 2007;447:92-96.

15 Al-Ghouleh A, Johal R, Sharquie IK, Emara M, Harrington H, Shakib F, et al: The glycosylation pattern of common allergens: the recognition and uptake of Der $\mathrm{p} 1$ by epithelial and dendritic cells is carbohydrate dependent. PLoS One 2012;7:e33929.

16 Kirkland TN, Finley F, Orsborn KI, Galgiani $\mathrm{JN}$ : Evaluation of the proline-rich antigen of Coccidioides immitis as a vaccine candidate in mice. Infect Immun 1998;66:3519-3522.
17 Banerjee S, Weber M, Blatt K, Swoboda I, Focke-Tejkl M, Valent $\mathrm{P}$, et al: Conversion of Der p 23, a new major house dust mite allergen, into a hypoallergenic vaccine. J Immunol 2014;192:4867-4875.

18 Jacquet A: The role of innate immunity activation in house dust mite allergy. Trends $\mathrm{Mol}$ Med 2011;17:604-611.

19 Shen Z: A type I peritrophic matrix protein from the malaria vector Anopheles gambiae binds to chitin. J Biol Chem 1998;273:1766517670.

20 Wijffels G, Eisemann C, Riding G, Pearson R, Jones A, Willadsen P, Tellam R: Novel family of chitin-binding proteins from insect type 2 peritrophic matrix. cDNA sequences, chitin binding activity, and cellular localization. J Biol Chem 2001;276:15527-15536.
21 Zakzuk J, Benedetti I, Fernández-Caldas E, Caraballo L: The influence of chitin on the immune response to the house dust mite allergen Blo t 12. Int Arch Allergy Immunol 2014; 163:119-129.

22 Chan T-F, Ji K-M, Yim AK-Y, Liu X-Y, Zhou J-W, Li R-Q, et al: The draft genome, transcriptome, and microbiome of Dermatophagoides farinae reveal a broad spectrum of dust mite allergens. J Allergy Clin Immunol 2015; 135:539-548.

23 Salazar F, Sewell HF, Shakib F, Ghaemmaghami AM: The role of lectins in allergic sensitization and allergic disease. J Allergy Clin Immunol 2013;132:27-36.

24 McCall C, Hunter S, Stedman K, Weber E, Hillier A, Bozic C, et al: Characterization and cloning of a major high molecular weight house dust mite allergen (Der f 15) for dogs. Vet Immunol Immunopathol 2001;78:231247. 